Association for Information Systems AIS Electronic Library (AISeL)

Wirtschaftsinformatik Proceedings 1999

Wirtschaftsinformatik

February 1999

\title{
Umsatzsteigerung durch Internet-Engagement?
}

Karl Kurbel

Universität Frankfurt/Oder, kurbel@euv-frankfurt-o.de

Frank Teuteberg

Universität Frankfurt/Oder, teute@euv-frankfurt-o.de

Follow this and additional works at: http://aisel.aisnet.org/wi1999

\section{Recommended Citation}

Kurbel, Karl and Teuteberg, Frank, "Umsatzsteigerung durch Internet-Engagement?" (1999). Wirtschaftsinformatik Proceedings 1999. 8.

http://aisel.aisnet.org/wi1999/8

This material is brought to you by the Wirtschaftsinformatik at AIS Electronic Library (AISeL). It has been accepted for inclusion in Wirtschaftsinformatik Proceedings 1999 by an authorized administrator of AIS Electronic Library (AISeL). For more information, please contact elibrary@aisnet.org. 


\section{Umsatzsteigerung durch Internet-Engagement?}

Karl Kurbel

Universität Frankfurt/Oder (kurbel@euv-frankfurt-o.de)

Frank Teuteberg

Universität Frankfurt/Oder (teute@euv-frankfurt-o.de)

\section{Inhalt}

1 Einführung

2 Charakterisierung der Unternehmen in der empirischen Untersuchung

3 Ursache-Wirkung-Beziehungen zwischen Nutzeffekten und Umsatzsteigerungen

3.1 Typen von Nutzeffekten

3.2 Zusammenhänge zwischen Nutzeffekten und Ableitung von

Wirkungsketten

3.3 Schlußfolgerungen

4 Ausgewählte Ergebnisse multivariater Analysen

4.1 Zusammenhang zwischen Wirtschaftszweig und Nutzeffekten

4.2 Zusammenhang zwischen Wirtschaftszweig und Umsatzsteigerung

4.3 Auswirkungen der Struktur von WWW-Anwendungen

4.3.1 Zusammenhang zwischen Kategorien und Umsatzsteigerung

4.3.2 Zusammenhang zwischen Kategorien und Nutzeffekten

5 Zusammenfassung 


\begin{abstract}
Vom Aufbau einer Internet-Präsenz versprechen sich Unternehmen meist betriebswirtschaftliche Vorteile. In dem Beitrag wird überprüft, inwieweit solche Vorteile empirisch nachweisbar sind. Die Grundlage bildet eine empirische Untersuchung deutscher Unternehmen im Internet. Über die an anderer Stelle beschriebenen deskriptiven Auswertungen hinaus wird versucht, Ursache-Wirkung-Zusammenhänge aufzudecken. Dazu werden die von den Unternehmen genannten Nutzeffekte analysiert und zu Wirkungsketten zusammengefaßt.
\end{abstract}

Besondere Aufmerksamkeit kommt den erzielten Umsatzsteigerungen zu. Es wird untersucht, inwieweit monetäre Auswirkungen den Nutzeffekten zugerechnet werden können. In ausgewählten multivariaten Analysen werden Unterschiede zwischen Unternehmen des produzierenden Sektors einerseits und des Dienstleistungs- und Handelssektors andererseits herausgearbeitet. Ebenso wird überprüft, ob durch intensiveres Internet-Engagement stärkere Nutzen- und Umsatzwirkungen erzielt werden. Insgesamt kann auf Basis des empirischen Datenmaterials gezeigt werden, daß Unternehmen, die im Internet präsent sind, zahlreiche Nutzeffekte realisieren, die über Wirkungsketten zu Umsatzsteigerungen führen.

\title{
1 Einführung
}

Viele Unternehmen haben in den letzten Jahren ihre Internet-Präsenz realisiert. Sie versprechen sich betriebswirtschaftliche Vorteile oder folgen einem Trend, der als zukunftsweisend angesehen wird und zumindest das Vermeiden zukünftiger Nachteile erwarten läßt. Mit diesem Beitrag wird das Ziel verfolgt, die vermuteten Wirkungen eines Internet-Engagements anhand emprischer Beobachtungen zu überprüfen und Schlußfolgerungen abzuleiten, die die Vorteilhaftigkeit belegen. Die Grundlage bildet eine empirische Untersuchung über den Stand der betrieblichen Internet-Nutzung in der Bundesrepublik Deutschland.

Über die deskriptiven Datenauswertungen hinaus, die an verschiedenen Stellen bereits veröffentlicht wurden (vgl. z.B. Kurbel/Teuteberg 1998; Kurbel 1998b; Teuteberg 1998), soll nachfolgend versucht werden, Zusammenhänge zwischen einzelnen Variablen unter Einsatz statistischer Analyseverfahren aufzudecken. $\mathrm{Zu}$ den wichtigsten Fragestellungen gehören das Aufzeigen des Zusammenhangs zwischen realisierten Nutzeffekten und Umsatzsteigerungen, das Ableiten von Nutzeffektketten, das Identifizieren größerer Wirkungsbereiche von Nutzeffekten sowie Analysen zum Zusammenhang zwischen der Intensität der Internet-Nutzung und möglichen Umsatzsteigerungen. 


\section{Charakterisierung der Unternehmen in der empirischen Untersuchung}

Die zugrundeliegende Untersuchung wurde weitestgehend elektronisch durchgeführt. Etwa 6000 Unternehmen wurden per E-Mail gebeten, einen auf dem Server der Europa-Universität bereitgestellten Fragebogen online auszufüllen. 495 Unternehmen $(8,25 \%)$ folgten dieser Aufforderung. 489 Fragebögen $(8,15 \%)$ enthielten sinnvoll interpretierbare Angaben.

Die Untersuchungseinheiten wurden Firmenverzeichnissen im World Wide Web entnommen. Aufgrund dieses Ansatzes zur Erzeugung der Stichprobe und der gezielten Aufforderung zur Teilnahme konnten einige Nachteile, die bei anonymen Online-Erhebungen häufig entstehen (Rücklaufquote, Datenqualität, Repräsentativität etc.) gemindert werden.

Bezüglich der Branchenzugehörigkeit wurde die Annahme getroffen, daß sich die Verteilung der im Internet präsenten Unternehmen über die Wirtschaftszweige hinweg in etwa in den Firmenverzeichnissen widerspiegelt, die im WWW verfügbar sind. Branchen, die im Internet und somit auch in den Verzeichnissen stärker vertreten sind, gelangten mit höherer Wahrscheinlichkeit in die Auswahl der angeschriebenen Unternehmen.

Die Zusammensetzung und die Größe der Stichprobe lassen Rückschlüsse auf das Internet-Engagement deutscher Unternehmen zu. Kritisch ist jedoch anzumerken, daß Repräsentativität der Stichprobe in Bezug auf Unternehmensgröße, Branche, Standort etc. nicht explizit angestrebt wurde. Mangels genauer Kenntnis der Grundgesamtheit aller im Internet vertretenen deutschen Unternehmen wäre dies nicht möglich gewesen.

Die Verteilung der Unternehmen hinsichtlich der Anzahl der Beschäftigten ist wie folgt:

\begin{tabular}{|c|c|c|c|c|c|c|}
\hline $\begin{array}{c}\text { Kategorie } \\
\text { (Anzahl Beschäftigte) }\end{array}$ & $1-10$ & $11-50$ & $51-100$ & $101-500$ & $501-1000$ & $>1000$ \\
\hline Anteil Unternehmen (\%) & 57,9 & 5,7 & 1,6 & 24,3 & 5,5 & 2,0 \\
\hline
\end{tabular}

Tabelle 1: Verteilung der Unternehmen nach Anzahl der Beschäftigten

Gegenüber der tatsächlichen Verteilung der deutschen Unternehmen sind Kleinbetriebe etwas unter- und größere Betriebe etwas überrepräsentiert. Nach den Daten des Statistischen Bundesamts haben $87 \%$ der deutschen Unternehmen weniger als 10 und 0,2 \% der Unternehmen mehr als 500 Mitarbeiter (vgl. Statistisches Bundesamt 1997, S. 32 ff.). 


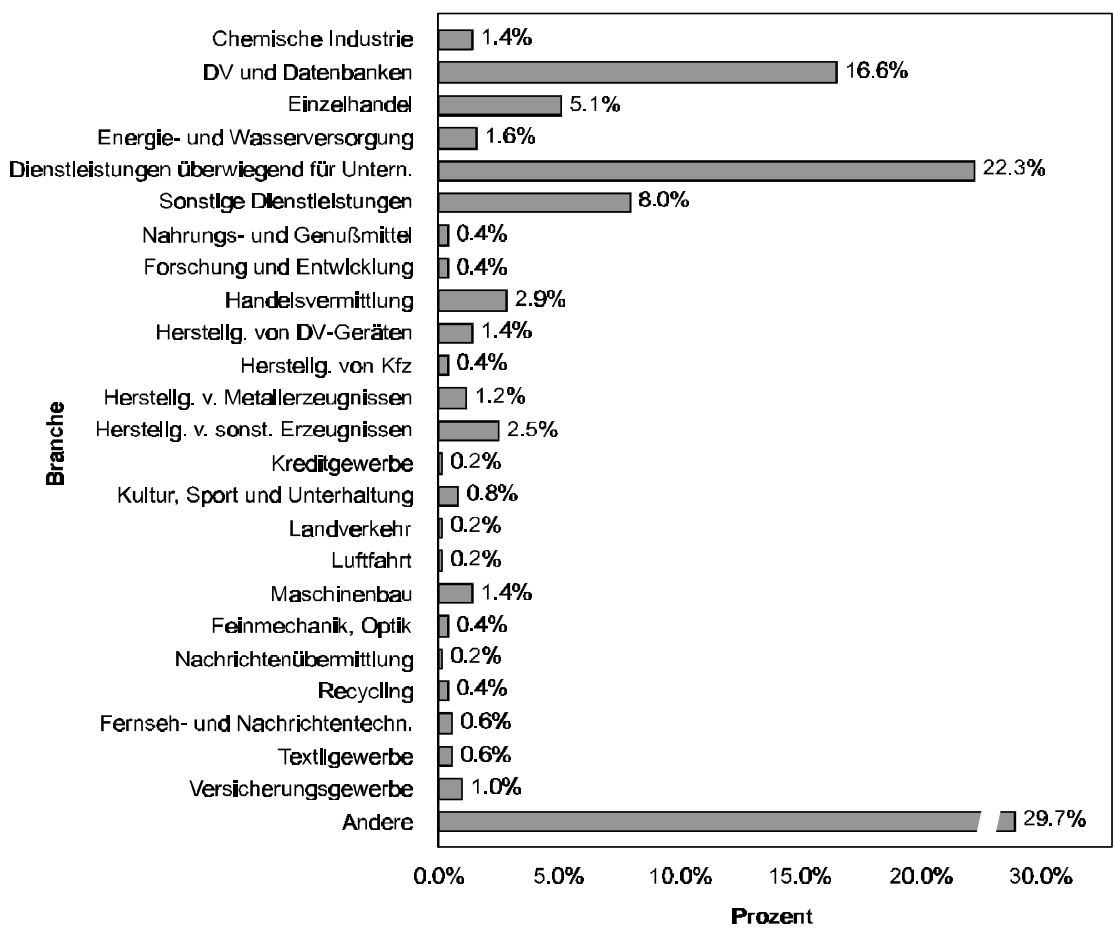

Abbildung 1: Verteilung der Branchen in der empirischen Untersuchung

Die Branchenzugehörigkeit der antwortenden Unternehmen wurde nach den Hauptkategorien des Statistischen Bundesamts gegliedert. Daß die dort verwendete Einteilung die heutigen Gegebenheiten nicht mehr angemessen widerspiegelt, zeigt sich nicht zuletzt daran, daß 29,7 \% der Unternehmen sich nur der Rubrik "Andere" zuordnen konnten. Dennoch läßt sie gewisse Schwerpunkte der InternetNutzung erkennen (vgl. Abbildung 1).

Ein deutliches Übergewicht weist der Dienstleistungssektor auf. Die Wirtschaftszweige "Erbringung von Dienstleistungen überwiegend für Unternehmen" mit 22,3\% und "Erbringung von sonstigen Dienstleistungen" mit 8,0\% machen zusammen über $30 \%$ des Fragebogenrücklaufs aus. Daneben fallen noch der Zweig "Datenverarbeitung und Datenbanken" mit 16,6 \% sowie der "Einzelhandel" mit 5,1 \% ins Gewicht. Diejenigen Unternehmen, die "Andere" angaben, nannten größtenteils Entwicklung, Vertrieb und Marketing von Hardware oder Software und Internet-Dienstleistungen im Bereich Grafik, Werbung und Marketing. 


\section{Ursache-Wirkung-Beziehungen zwischen Nutzeffekten und Umsatzsteigerungen}

\subsection{Typen von Nutzeffekten}

Im Bereich der Internet-Nutzung sind Nutzenbewertungen schwierig. Dies liegt zum einen daran, daß sich der kommerzielle Einsatz des Internet noch in einer frühen Phase befindet und somit wenig empirische Vergleichswerte vorliegen. Zum anderen sind die Nutzeffekte äußerst vielschichtig und größtenteils nicht direkt monetär bewertbar. Wenn man monetäre Aussagen machen will, müssen diese auf indirektem Wege abgeleitet werden.

Nutzeffekte lassen sich über ein Geflecht von Wirkungsbeziehungen in Form von Wirkungs- oder Nutzeffektketten in Verbindung bringen. Dies wurde von anderen Autoren im Bereich der Informationsverarbeitung verschiedentlich praktiziert (vgl. z.B. Schumann 1992b; Linß 1995 S. 69 ff.; Schumann/Mertens 1990). Teilweise können sie zu übergeordneten Effekten aggregiert werden, wenn man größere Wirkungsbereiche identifizieren will. Zum Beispiel lassen sich Nutzeffekte bezüglich ihrer Wirkungsbereiche verschiedenen Ebenen zuordnen. Schumann unterscheidet Nutzeffekte auf Arbeitsplatzebene, Abteilungs-/Bereichsebene, Unternehmensebene sowie auf zwischenbetrieblicher Ebene oder Marktebene (vgl. Schumann 1992a, S. 63 f.).

Nach dem Kriterium Bewertbarkeit lassen sich Nutzeffekte in monetär quantitative, nicht-monetär quantitative und qualitative Nutzeffekte unterscheiden (vgl. Linß 1995, S. 33 f.). Um die qualitativen sowie nicht-monetär quantitativen Nutzeffekte indirekt zu monetarisieren, werden nachfolgend Wirkungsketten abgeleitet. Dies wird vor allem mit Blick auf realisierte Umsatzsteigerungen durchgeführt. Außerdem werden diejenigen Nutzeffekte identifiziert, die weitere Effekte, auf unterschiedlichen Unternehmensebenen, nach sich ziehen und somit einen größeren Wirkungsbereich haben.

\subsection{Zusammenhänge zwischen Nutzeffekten und Ableitung von Wirkungsketten}

Um Zusammenhänge zwischen den Nutzeffekten herstellen zu können, werden bivariate Korrelationsanalysen auf der Grundlage von Kendall's $\tau_{\mathrm{B}}$ durchgeführt. Diese ermöglichen jedoch noch keine Aussage darüber, welcher Nutzeffekt als verursachend für einen anderen Nutzeffekt anzusehen ist. Bei der Interpretation der Ergebnisse sind daher Ursache-Wirkung-Beziehungen aus Plausibilitätsüberlegungen abzuleiten.

Darüber hinaus ist einschränkend anzumerken, daß Abhängigkeiten zwischen einzelnen Ursache-Wirkung-Beziehungen durch die Korrelationsanalysen nicht erfaßt werden. So ist es möglich, daß zwei Nutzeffekte, die untereinander in einem 
Ursache-Wirkung-Zusammenhang stehen, gemeinsam wiederum zu einem oder mehreren weiteren Nutzeffekten eine Ursache-Wirkung-Beziehung aufweisen.

Von den 43 Nutzeffekten, die im Fragebogen vorgegeben waren, wurden die folgenden 15 am häufigsten genannt:

Image verbessert

Kundenservice erhöht

Neue Geschäftspartner gewonnen

Datenübertragung in Papierform entfällt

Differenzierung im Wettbewerb

Räumliche Grenzen überwunden

Schnelleres und flexibleres Reagieren auf Kundenwünsche ermöglicht

Neue Märkte erschlossen

Umfassendere Informationsbasis realisiert

Kundenzufriedenheit erhöht

Abläufe vereinfacht

Arbeitszeit eingespart

Marktvorteile erreicht

Kundenbindung erhöht

Flexibilität erhöht

Tabelle 2 zeigt die Stärke der statistischen Beziehung zwischen jeweils zwei Nutzeffekten. Zur Berechnung der Maßzahlen wurden die Zahl k der konkordanten (gleichgerichteten) und die Zahl d der diskordanten (entgegengesetzt gerichteten) Paare von Nutzeffekten herangezogen. Ein Paar von Nutzeffekten (z.B. "Kundenservice erhöht" und "Image verbessert") wird als konkordant bezeichnet, wenn beide Nutzeffekte gleichzeitig realisiert werden. Kann nur einer von beiden beobachtet werden, so ist das Paar diskordant.

Kendall's $\tau_{\mathrm{B}}$ wurde herangezogen, um Aussagen über die Stärke des Zusammenhangs zwischen Nutzeffekten zu gewinnen. Die Maßzahl ist so angelegt, daß sie Werte zwischen -1 und +1 erreichen kann, wobei größere Absolutwerte auf stärkere Zusammenhänge hindeuten. Sie ist definiert als (vgl. Janssen/Laatz 1997, S. 233):

$$
\tau_{\mathrm{B}}=\frac{\mathrm{k}-\mathrm{d}}{\sqrt{\left(\mathrm{k}+\mathrm{d}+\mathrm{t}_{\mathrm{x}}\right) \cdot\left(\mathrm{k}+\mathrm{d}+\mathrm{t}_{\mathrm{y}}\right)}}
$$

Dabei ist $t_{x}$ die Zahl der Paare, bei denen auf Nutzeffekt $x$ eine Bindung vorliegt, und $t_{y}$ die Zahl der Paare, bei denen auf Nutzeffekt y eine Bindung vorliegt. Ein Paar ist gebunden, wenn die Werte der beiden Variablen gleich sind. Falls von allen 489 Unternehmen ein bestimmtes Paar von Nutzeffekten stets gleichzeitig realisiert wurde, d.h. $\mathrm{k}=489$ und $\mathrm{d}=0$, so nimmt Kendall's $\tau_{\mathrm{B}}$ den Wert 1 an (totaler positiver Zusammenhang). Die Werte in Tabelle 2 stellen somit ein Maß für die Stärke der Beziehungen zwischen den Nutzeffekten dar. Werte von 0,30 oder größer wurden fett markiert. In diesen Fällen besteht ein statistisch signifikanter Zusammenhang zwischen den jeweiligen Nutzeffekten bei einem Signifikanzniveau von 0,05 . 


\begin{tabular}{|c|c|c|c|c|c|c|c|c|c|c|c|c|c|c|c|}
\hline 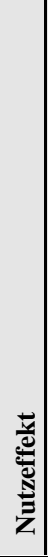 & 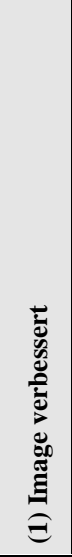 & 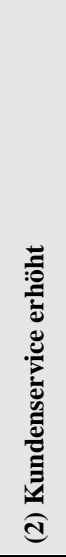 & 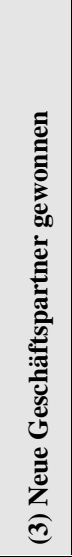 & 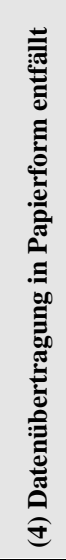 & 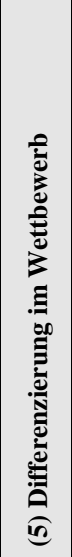 & 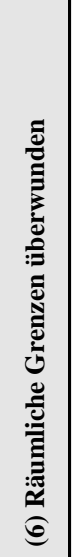 & 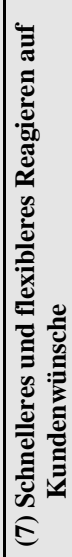 & 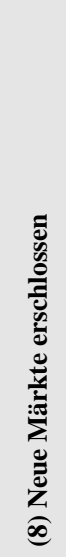 & 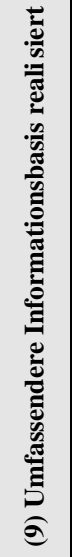 & 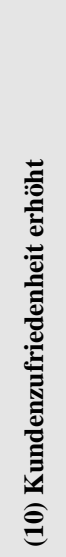 & 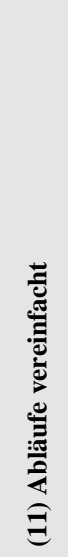 & 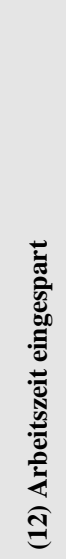 & 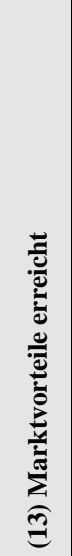 & 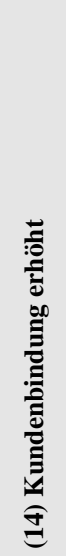 & 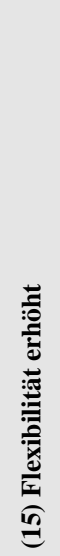 \\
\hline (1) & 00 & & 2 & 0,19 & 0,37 & 18 & 0,22 & 0,23 & 0,22 & 0,24 & 0,07 & 0,12 & 0,28 & 30 & 0,17 \\
\hline (2) & 0,31 & 00 & 20 & 0,24 & $\mathbf{0 , 3 3}$ & 0,22 & $\mathbf{0 , 3 5}$ & 0,23 & $\mathbf{0 , 3 3}$ & 0,44 & 0,22 & 0,11 & $\mathbf{0 , 3 1}$ & 0,48 & 0,27 \\
\hline (3) & 22 & 20 & 00 & 0,26 & 27 & 40 & 0,24 & 0,51 & 0,26 &, 24 & 0,23 & 0,19 &, 40 & 0,28 & 0,29 \\
\hline (4) & 0.19 & 24 & 26 & 1,00 & 018 & 0,20 & 0,23 & 0,20 & 0,20 &, 22 & 0,45 & 32 &, 19 & 0,21 & 0,35 \\
\hline (5) & 0,37 & 33 & 27 & 0,18 & 1,00 & 0,26 & 0,24 & $\mathbf{0 , 3 0}$ & 0,28 & ,25 & 0,21 & 0,16 &, 33 & $\mathbf{0 , 3 5}$ & 0,25 \\
\hline (6) & 0,18 & & & 20 & 0,26 & & 3 & 0,29 & 0 & 0 & 20 & 9 & 38 & 7 & c \\
\hline (7) & 0,22 & 35 & 24 & 0,23 & 0,24 & 0,23 & 1,00 & 0,27 & $\mathbf{0 , 3 8}$ & 48 & 0,29 & 0,18 & ,30 & $\mathbf{0 , 3 8}$ & $\mathbf{0 , 3 0}$ \\
\hline (8) & 23 & & 51 & 0,20 & 0,30 & 29 & 0,27 & 1,00 & 0,21 & 30 & 0,19 & 0,14 &, 41 &, 30 & 0,23 \\
\hline (9) & 22 & & & 20 & 0,28 & 0 & 88 & 0,21 & 1,00 & 35 & 0,26 & 0,20 & ,35 &, 29 & $\mathbf{0 , 3 0}$ \\
\hline (10) & 0,24 & 44 & 33 & 0,22 & 0,25 & $\mathbf{0 , 3 0}$ & $\mathbf{0 , 4 8}$ & $\mathbf{0 , 3 0}$ & 0,35 & 1,00 & $\mathbf{0 , 3 1}$ & 0,24 & $\mathbf{0 , 4 4}$ & $\mathbf{0 , 4 7}$ & 0,36 \\
\hline (11) & 0,07 & 0,22 & 3 & 45 & $\mathbf{0}$ & 0 & 9 & 0,19 & 26 & 31 & 00 & 4 & 22 &, 23 & 0,38 \\
\hline (12) & 0,12 & 11 & 19 & $\mathbf{0 , 3 2}$ & 0,16 & 0,20 & 18 & 0,14 & 0,20 & 0,24 & 0,47 & 1,00 & 0,20 & 0,15 & 0,2 \\
\hline (13) & 0,28 & & $\mathbf{0 , 4 0}$ & 0,19 & $\mathbf{0 , 3 3}$ & $\mathbf{0 , 3 8}$ & $\mathbf{0 , 3 0}$ & 0,41 & 0,35 & 44 & 0,22 & 0,20 & 1,00 & $\mathbf{0 , 3 8}$ & $\mathbf{0 , 3 1}$ \\
\hline (14) & $\mathbf{0 , 3 0}$ & $\mathbf{0 , 4 0}$ & 0,28 & 0,21 & 0,35 & 0,17 & $\mathbf{0 , 3 8}$ & $\mathbf{0 , 3 0}$ & 29 &, 47 & 0,23 & 0,15 &, 38 & 1,00 & 0,31 \\
\hline 15) & 17 & 0,27 & & 35 & 0,25 & 25 & 0,30 & 0,23 & $\mathbf{0 , 3 0}$ & ,36 & 0,38 & 0,29 & 0,31 & 0,31 & 1,00 \\
\hline
\end{tabular}

Tabelle 2: Statistischer Zusammenhang zwischen einzelnen Nutzeffekten (Maßzahl Kendall's $\tau_{\mathbf{B}}$ ) 
Nach der Stärke des Zusammenhangs können die Nutzeffektpaare wie folgt unterschieden werden.

\section{Statistische Beziehung nach Kendall's $\tau_{\mathbf{B}} \geq \mathbf{0 , 3 0}$}

- Abläufe vereinfacht

$\Leftrightarrow \quad$ Flexibilität erhöht

- Datenübertragung in Papierform entfällt $\Leftrightarrow$

$\Leftrightarrow \quad$ Kundenzufriedenheit erhöht

- Differenzierung im Wettbewerb

$\Leftrightarrow \quad$ Flexibilität erhöht

- Flexibilität erhöht

$\Leftrightarrow \quad$ Marktvorteile erreicht

$\Leftrightarrow \quad$ Image verbessert

$\Leftrightarrow \quad$ Kundenbindung erhöht

$\Leftrightarrow \quad$ Kundenzufriedenheit erhöht

$\Leftrightarrow \quad$ Marktvorteile erreicht

$\Leftrightarrow \quad$ Schnelleres und flexibleres

Reagieren auf Kundenwün-

- Kundenbindung erhöht

sche ermöglicht

$\Leftrightarrow \quad$ Differenzierung im

Wettbewerb

$\Leftrightarrow \quad$ Image verbessert

- Kundenservice erhöht

$\Leftrightarrow \quad$ Marktvorteile erreicht

$\Leftrightarrow \quad$ Differenzierung im

Wettbewerb

$\Leftrightarrow \quad$ Marktvorteile erreicht

$\Leftrightarrow \quad$ Schnelleres und flexibleres

Reagieren auf Kundenwünsche ermöglicht

- Kundenzufriedenheit erhöht

$\Leftrightarrow \quad$ Image verbessert

- Neue Märkte erschlossen

$\Leftrightarrow \quad$ Neue Märkte erschlossen

$\Leftrightarrow \quad$ Kundenbindung erhöht

$\Leftrightarrow \quad$ Differenzierung im

Wettbewerb

- Räumliche Grenzen überwunden

$\Leftrightarrow \quad$ Kundenzufriedenheit erhöht

$\Leftrightarrow \quad$ Marktvorteile erreicht

- Schnelleres und flexibleres Reagieren auf Kundenwünsche ermöglicht

Kundenbindung erhöht

$\Leftrightarrow \quad$ Marktvorteile erreicht

$\Leftrightarrow \quad$ Umfassendere Informationsbasis realisiert

- Umfass. Informationsbasis realisiert

$\Leftrightarrow \quad$ Kundenservice erhöht

$\Leftrightarrow \quad$ Räumliche Grenzen über wunden

$\Leftrightarrow \quad$ Flexibilität erhöht

$\Leftrightarrow \quad$ Kundenzufriedenheit erhöht

$\Leftrightarrow \quad$ Marktvorteile erreicht 


\section{Statistische Beziehung nach Kendall's $\tau_{\mathbf{B}} \geq \mathbf{0 , 4 0}$}

- Datenübertragung in Papierform entfällt $\Leftrightarrow$

- Kundenservice erhöht

Abläufe vereinfacht

- Kundenzufriedenheit erhöht

$\Leftrightarrow \quad$ Kundenbindung erhöht

- Neue Geschäftspartner gewonnen

$\Leftrightarrow \quad$ Kundenzufriedenheit erhöht

$\Leftrightarrow \quad$ Kundenbindung erhöht

$\Leftrightarrow \quad$ Marktvorteile erreicht

$\Leftrightarrow \quad$ Marktvorteile erreicht

$\Leftrightarrow \quad$ Räumliche Grenzen

überwunden

- Schnelleres und flexibleres Reagieren $\Leftrightarrow \quad$ Kundenzufriedenheit erhöht auf Kundenwünsche ermöglicht

- Neue Märkte erschlossen $\quad \Leftrightarrow \quad$ Marktvorteile erreicht

\section{Statistische Beziehung nach Kendall's $\tau_{\mathbf{B}} \geq \mathbf{0 , 5 0}$}

- Neue Geschäftspartner gewonnen $\quad \Leftrightarrow \quad$ Neue Märkte erschlossen

Zur Ermittlung der Wirkungsketten werden die Vorgänger-Nachfolger-Beziehungen zwischen den Nutzeffekt-Paaren einzeln analysiert. Nimmt man z.B. das Paar "Kundenservice erhöht" und "Differenzierung im Wettbewerb", so ist offenbar eine Ursache-Wirkung-Beziehung derart, daß durch Erhöhung des Kundenservice eine Differenzierung von Wettbewerbern erreicht werden konnte, plausibler als die umgekehrte Annahme, daß sich aufgrund der Differenzierungsstrategie der Kundenservice verbessert hat. Auf diese Weise können einzelne Nutzeffekte in eine Reihenfolge gebracht und in Form einer Wirkungskette, mit Auswirkungen und Einflußfaktoren, dargestellt werden.

Nutzeffekte, die in ihrem Wirkungsbereich auf einen bestimmten Arbeitsplatz begrenzt sind, gehören nach der Schumann'schen Ebeneneinteilung zur Arbeitsplatzebene (vgl. Schumann 1992a, S. 63 f.). Ein Beispiel ist die Einsparung von Arbeitszeit, die z.B. dadurch erreicht wird, daß Recherchen vom PC-Arbeitsplatz aus im Internet durchgeführt werden können. Der Nutzeffekt "Arbeitszeit eingespart" wurde von den 489 Unternehmen relativ losgelöst von anderen Nutzeffekten beobachtet. Nur zu "Datenübertragung in Papierform entfällt" sowie "Abläufe vereinfacht" besteht ein statistischer Zusammenhang, der stärker als 0,30 ist. Es handelt sich offenbar um einen Nutzeffekt mit begrenztem Wirkungsbereich.

Nutzeffekte, bei denen weitergehende Abläufe oder Vorgänge über den einzelnen Arbeitsplatz hinaus betroffen sind, lassen sich der Abteilungs-/Bereichsebene zuordnen; d.h., das Internet-Engagement wirkt sich auf eine ganze Abteilung oder einen ganzen Unternehmensbereich aus. Ein Beispiel ist der Nutzeffekt "Umfassendere Informationsbasis realisiert".

Dieser Nutzeffekt steht in Zusammenhang mit Nutzeffekten auf der Unternehmensebene, d.h. mit Nutzeffekten, die sich auf das gesamte Unternehmen auswirken. $\mathrm{Zu}$ "Schnelleres und flexibleres Reagieren auf Kundenwünsche ermöglicht" und "Flexibilität erhöht" besteht ein statistischer Zusammenhang von 0,38 bzw. 0,30 . 
Nutzeffekte, die sich über die Unternehmensebene hinaus auswirken, liegen auf der zwischenbetrieblichen Ebene oder Marktebene. Sie treten insbesondere bei zwischenbetrieblich integrierten Systemen auf, mittels derer Beziehungen zu Kunden, Lieferanten oder anderen Unternehmen hergestellt werden. Auf der zwischenbetrieblichen Ebene bzw. Marktebene sind Nutzeffekte zu beobachten, die zusammen mit dem auf Unternehmensebene angesiedelten Effekt "Umfassendere Informationsbasis realisiert" vorkommen. Zwischen den Nutzeffekten "Räumliche Grenzen überwunden" (0,30), "Kundenservice erhöht" $(0,33)$, Kundenzufriedenheit erhöht" $(0,35)$ sowie "Marktvorteile erreicht" $(0,35)$ und dem Effekt "Umfassendere Informationsbasis realisiert" besteht ein statistischer Zusammenhang von größer als 0,30 .

Betrachtet man die anderen Nutzeffekte, so zeigt sich etwa, daß der Effekt "Flexibilität erhöht" zu sieben weiteren Nutzeffekten, z.B. zu "Schnelleres Reagieren auf Kundenwünsche ermöglicht", "Kundenzufriedenheit erhöht" und "Marktvorteile erreicht", in einem engeren Zusammenhang steht (größer als 0,30).

Relativ isoliert tritt der allgemeine Nutzeffekt "Image verbessert" auf. Lediglich "Kundenservice erhöht" sowie "Differenzierung im Wettbewerb" weisen mit 0,31 bzw. 0,37 einen statistischen Zusammenhang von größer als 0,30 mit diesem Effekt auf. Offenbar kann die Imageverbesserung auch erreicht werden, ohne daß andere Nutzeffekte eintreten.

Tabelle 2 verdeutlicht, daß ein Internet-Engagement sowohl stark wettbewerbsrelevante Wirkungen als auch Einfluß auf die Zufriedenheit der Kunden hat. So stehen jeweils zehn der am häufigsten genannten Nutzeffekte mit den Nutzeffekten "Marktvorteile erreicht" sowie "Kundenzufriedenheit erhöht" in einem stärkeren Zusammenhang als 0,30. Diese beiden Nutzeffekte resultieren größtenteils aus den anderen zehn Nutzeffekten, wie man durch Analyse der einzelnen UrsacheWirkung-Beziehungen zeigen kann. (Zum Beispiel verbessert eine Erhöhung der Lieferflexibilität die Kundenzufriedenheit und nicht umgekehrt.)

Um mögliche Wirkungsketten, die sich aus Tabelle 2 ableiten lassen, monetarisieren zu können, sollen die Auswirkungen auf den Umsatz der Unternehmen analysiert werden.

In der Erhebung beantworteten $21 \%$ der Unternehmen die Frage "Konnte durch die Nutzung des Internet eine Umsatzsteigerung erreicht werden?" mit "ja" und $19,9 \%$ mit "nein". 57,5 \% stellten fest, daß eine Einschätzung noch nicht möglich sei. Die meisten der 103 Unternehmen, die eine Umsatzsteigerung realisierten, waren allerdings nicht in der Lage, den Zuwachs genauer zu quantifizieren. Der Rest nannte überwiegend Größenordnungen unter $20 \%$ (vgl. Abbildung 2).

Tabelle 3 stellt die prozentualen Anteile derjenigen Nutzeffekte dar, die von den Unternehmen mit Umsatzsteigerung durch Internet-Engagement realisiert (1) bzw. nicht realisiert (2) wurden. In der Spalte "Ohne Einschätzung" sind die prozentualen Anteile der Nutzeffekte aufgeführt, die von denjenigen Unternehmen beobachtet wurden, welche noch keine Angaben bezüglich einer Umsatzsteigerung machen konnten. 
Von besonderer Bedeutung ist die Relation aus (1) und (2), wie in der rechten Spalte der Tabelle angegeben. So wurde z.B. der relativ allgemeine Nutzeffekt "Image verbessert" von 76,1 \% derjenigen Unternehmen beobachtet, die eine Umsatzsteigerung erreichten, aber auch von 55,6 \% der Unternehmen, die keine Umsatzsteigerung realisieren konnten. Angesichts einer Relation von 1,4 : 1 kann dieser Effekt kaum als "Schlüsselfaktor" für Umsatzzuwächse angesehen werden.

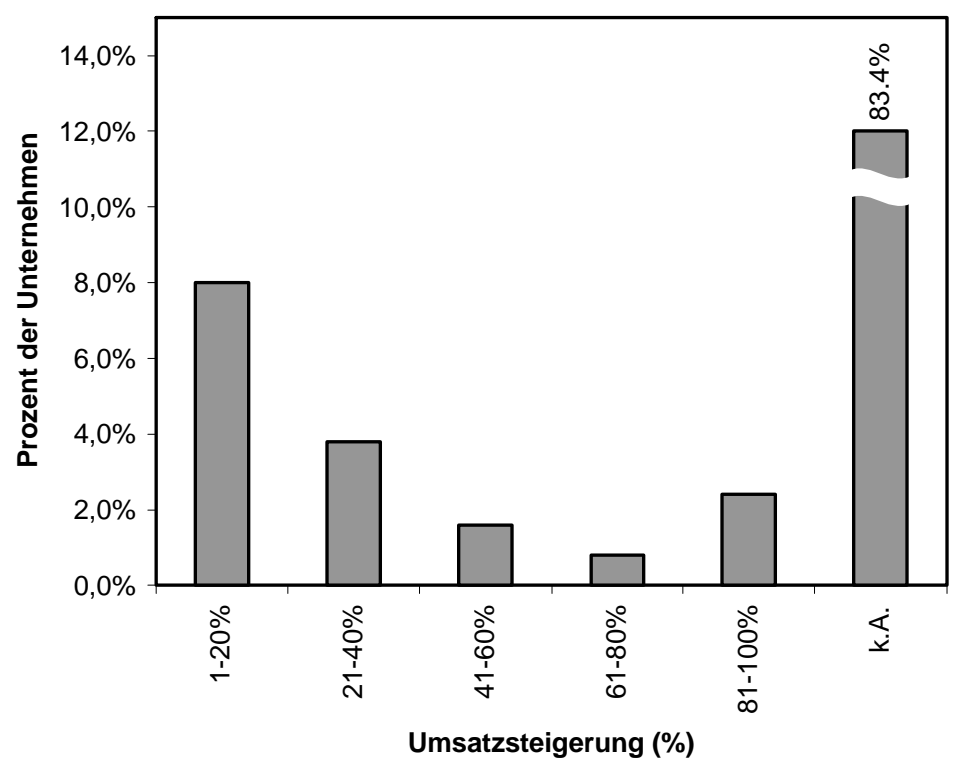

Abbildung 2: Beobachtete Umsatzsteigerung (Basis: 103 Unternehmen)

Im folgenden soll untersucht werden, ob die zuvor hervorgehobenen Nutzeffekte "Marktvorteile erreicht" sowie "Kundenzufriedenheit erhöht" letztlich zu einer Umsatzsteigerung führten. Damit ließe sich eine Monetarisierung der Nutzeffektketten vornehmen. Es zeigt sich, daß 62,8 \% der Unternehmen, die Marktvorteile erzielten, auch eine Umsatzsteigerung realisieren konnten, während nur 9,5\% der Unternehmen, die diesen Nutzeffekt beobachten, keine Umsatzsteigerung verzeichneten. Mit einer Relation von 4,1 : 1 ist der Nutzeffekt "Kundenzufriedenheit erhöht" offensichtlich einer, der als ursächlich für eine Umsatzsteigerung anzusehen ist.

Insgesamt kann man sagen, daß Unternehmen, die Umsatzzuwächse erzielten, gleichzeitig sehr häufig die markt- und kundenorientierten Nutzeffekte beobachteten. Aber auch Nutzeffekte, die weitaus seltener realisiert wurden, zeigen teilweise ein starkes Potential für Umsatzsteigerungen. Zum Beispiel wurde der Nutzeffekt "Lieferflexibilität erhöht" fast nur von solchen Unternehmen beobachtet, bei denen auch der Umsatz anwuchs (Relation 10,5 : 1). Offenbar gehen mit WWWAngeboten, die auf eine stärkere Lieferflexibilität abzielen, überdurchschnittlich 
häufig Umsatzwirkungen einher. Der Effekt "Lieferflexibilität erhöht" kann somit durchaus als ein Schlüsselfaktor für Umsatzsteigerungen angesehen werden.

\begin{tabular}{|c|c|c|c|c|}
\hline Realisierte Nutzeffekte & $\begin{array}{l}\text { Umsatz- } \\
\text { steig. } \\
\text { realisiert } \\
(1)\end{array}$ & $\begin{array}{l}\text { Umsatz- } \\
\text { steig. } \\
\text { nicht } \\
\text { realisiert } \\
\text { (2) }\end{array}$ & $\begin{array}{c}\text { Ohne } \\
\text { Einschät- } \\
\text { zung }\end{array}$ & $\begin{array}{l}\text { Relation } \\
(1):(2)\end{array}$ \\
\hline Neue Geschäftspartner gewonnen & 77,9 & 22,2 & 49,5 & $3,5: 1$ \\
\hline Image verbessert & 76,1 & 55,6 & 70,8 & $1,4: 1$ \\
\hline Datenübertragung in Papierform entfällt & 66,4 & 33,3 & 47,3 & $2,0: 1$ \\
\hline Kundenservice erhöht & 66,4 & 36,5 & 52 & $1,8: 1$ \\
\hline Marktvorteile erreicht & 62,8 & 9,5 & 30,2 & $6,6: 1$ \\
\hline Neue Märkte erschlossen & 61,1 & 14,3 & 38,1 & $4,3: 1$ \\
\hline Schnelleres Reagieren auf Kundenwünsche & 58,4 & 19,0 & 39,5 & $3,1: 1$ \\
\hline Räumliche Grenzen überwunden & 55,8 & 27,0 & 42,7 & $2,1: 1$ \\
\hline Kundenbindung erhöht & 54,0 & 14,3 & 33,1 & $3,8: 1$ \\
\hline Engere Bindung der Partner realisiert & 53,1 & 7,9 & 28,1 & $6,7: 1$ \\
\hline Arbeitszeit eingespart & 52,2 & 15,9 & 35,2 & $3,3: 1$ \\
\hline Differenzierung im Wettbewerb & 52,2 & 28,6 & 44,8 & $1,8: 1$ \\
\hline Kundenzufriedenheit erhöht & 52,2 & 12,7 & 35,2 & $4,1: 1$ \\
\hline Flexibilität erhöht & 51,3 & 14,3 & 33,1 & $3,6: 1$ \\
\hline Abläufe vereinfacht & 50,4 & 20,6 & 36,3 & $2,5: 1$ \\
\hline Umfassendere Informationsbasis realisiert & 46,0 & 28,6 & 38,1 & $1,6: 1$ \\
\hline Reaktionsfähigkeit des Untern. erhöht & 38,9 & 15,9 & 35,2 & $2,5: 1$ \\
\hline Wartezeit verkürzt & 36,3 & 14,3 & 23,1 & $2,5: 1$ \\
\hline Durchlaufzeit verkürzt & 35,4 & 11,1 & 21,4 & $3,2: 1$ \\
\hline Papierflut im Unternehmen eingedämmt & 35,4 & 11,1 & 17,1 & $3,2: 1$ \\
\hline Allgemeine Kostensenkung realisiert & 28,3 & 3,2 & 10,3 & $8,8: 1$ \\
\hline Engere zwischenbetriebliche Zusammenarbeit & 23,9 & 6,3 & 15,3 & $3,8: 1$ \\
\hline Aus-/Weiterbildung extern verbessert & 22,1 & 6,3 & 16,7 & $3,5: 1$ \\
\hline Verwaltungsaufwand reduziert & 22,1 & 7,9 & 9,3 & $2,8: 1$ \\
\hline Wissensmultiplikation realisiert & 22,1 & 15,9 & 15,7 & $1,4: 1$ \\
\hline $\begin{array}{l}\text { Schnellere Zugriffsmöglichkeiten auf betriebswirt- } \\
\text { schaftl. relevante Daten realisiert }\end{array}$ & 21,2 & 3,2 & 11,7 & $6,6: 1$ \\
\hline Einmalig Datenerfassung ermöglicht & 20,4 & 6,3 & 12,8 & $3,2: 1$ \\
\hline Routinetätigkeiten verringert & 20,4 & 4,8 & 10,0 & $4,3: 1$ \\
\hline Transportzeit minimiert & 17,7 & 3,2 & 5,3 & $5,5: 1$ \\
\hline Lieferflexibilität erhöht & 16,8 & 1,6 & 5,0 & $10,5: 1$ \\
\hline Logistikkosten gesenkt & 16,8 & 0,0 & 4,6 & $16,8: 0$ \\
\hline Stärkere Einflußnahme auf andere Unternehmen & 16,8 & 4,8 & 7,5 & $3,5: 1$ \\
\hline Versandabwicklung beschleunigt & 15,9 & 1,6 & 6,0 & 9,9:1 \\
\hline Parallelisierung von Vorgängen ermöglicht & 15,9 & 1,6 & 6,0 & $9,9: 1$ \\
\hline Produktkosten gesenkt & 15,0 & 0,0 & 2,5 & $15: 0$ \\
\hline Lieferbereitschaft erhöht & 14,2 & 3,2 & 3,2 & $4,4: 1$ \\
\hline Personalproduktivität erhöht & 14,2 & 1,6 & 8,5 & $8,9: 1$ \\
\hline Transportkosten verringert & 14,2 & 1,6 & 7,1 & $8,9: 1$ \\
\hline Funktionen zusammengefaßt & 13,3 & 1,6 & 5,7 & $8,3: 1$ \\
\hline Raumkosten reduziert & 12,4 & 1,6 & 5,0 & $7,8: 1$ \\
\hline Fertigungskapazität erhöht & 8,8 & 1,6 & 1,8 & $5,5: 1$ \\
\hline Gutes Personal gehalten & 6,2 & 1,6 & 1,8 & $3,9: 1$ \\
\hline Just-in-time-Produktion ermöglicht & 5,3 & 1,6 & 2,5 & $3,3: 1$ \\
\hline
\end{tabular}

Tabelle 3: Verteilung der Nutzeffekte in Abhängigkeit von Umsatzsteigerungen (\%) 


\subsection{Schlußfolgerungen}

Auf Basis der vorstehenden Analysen konnten diejenigen Nutzeffekte herausgearbeitet werden, die sich indirekt auf den Umsatz der Unternehmen auswirkten. Dazu gehören Nutzeffekte wie "Marktvorteile erreicht", "Neue Märkte erschlossen", "Kundenbindung erhöht", "Kundenzufriedenheit erhöht", und "Engere Bindung der Partner realisiert". Es wurde deutlich, daß das Internet eine starke wettbewerbsrelevante Wirkung hat und daß kundenbezogene Faktoren eine bedeutende Rolle spielen. Zwölf der fünfzehn häufigsten Nutzeffekte stehen in engem Zusammenhang mit "Marktvorteile erreicht" sowie "Kundenzufriedenheit erhöht".

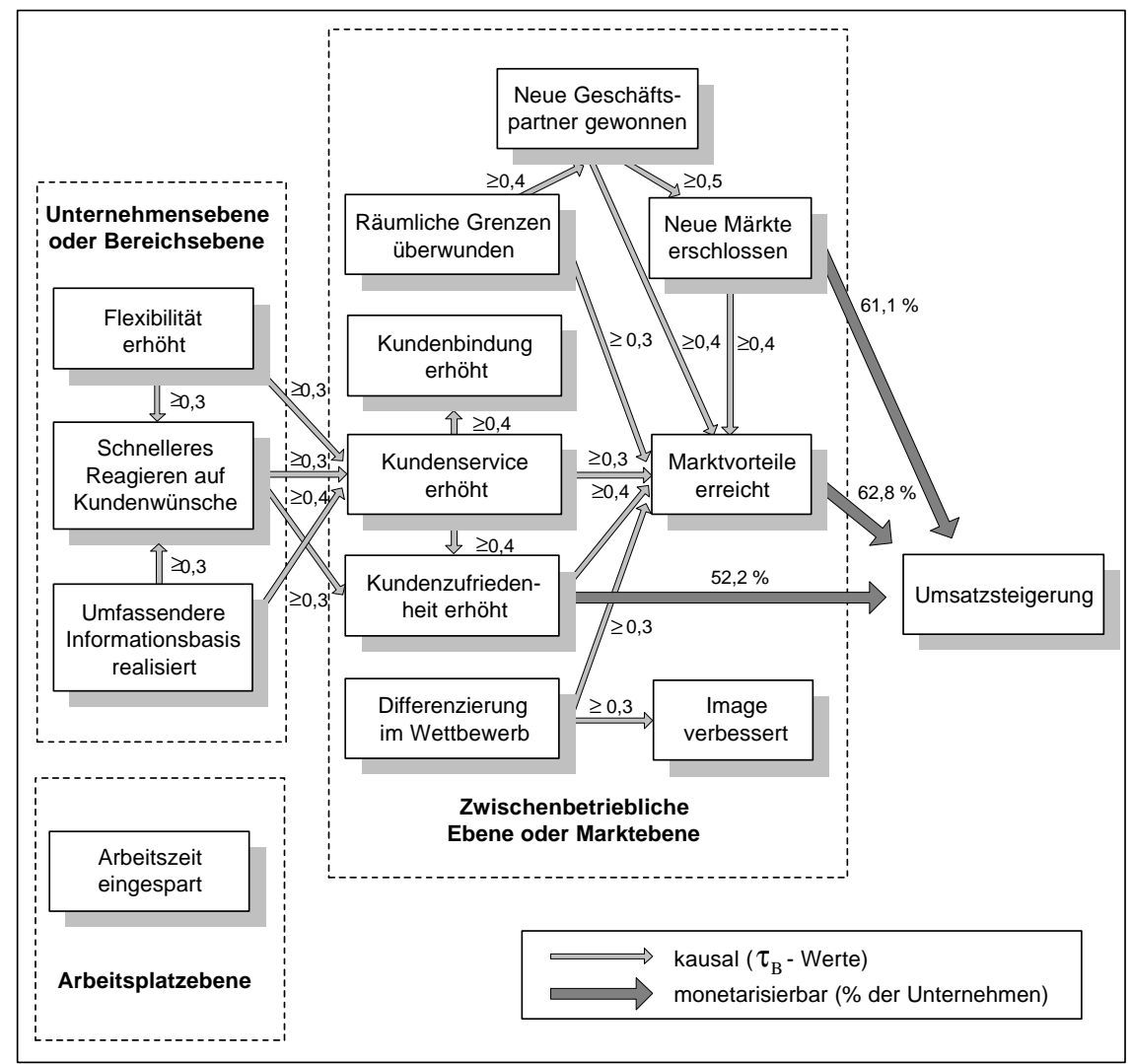

Abbildung 3: Wirkungskette

Durch Analyse und Interpretation der Beziehungen zwischen den Nutzeffekten kann man hypothetische Wirkungsketten in einem Modell formulieren. Abbildung 3 zeigt ein solches Modell, das unter Zuhilfenahme der Analysen und empirischen Ergebnisse aus den Tabellen 1 und 2 validiert wurde. Da es schwierig ist, hinreichend realistische Modelle von Wirkungsketten vorab, d.h. ohne Betrachtung der empirischen Befunde, aufzustellen, bleibt nur der Weg, die Signifikanz im nach- 
hinein zu testen. Es ist anzunehmen, daß weitergehende Analysen und Verbesserungen gegenüber unserer Vorgehensweise, die sich primär auf Korrelationsanalysen und Plausibilitätsüberlegungen stützt, auf der Basis von Verfahren wie "Path Analysis" bzw. "Structural Equation Modeling" (vgl. Anderson/Gerbing 1988, S. 411 ff.) möglich wären.

Die Effekte auf zwischenbetrieblicher Ebene oder Marktebene sind teilweise indirekte Effekte (z.B. "Kundenservice erhöht", "Marktvorteile erreicht", "Image verbessert", "Neue Märkte erschlossen", "Differenzierung im Wettbewerb"), die aus Effekten auf Unternehmens- oder Bereichsebene (z.B. "Schnelleres und flexibleres Reagieren auf Kundenwünsche ermöglicht") resultieren.

Abbildung 3 zeigt darüber hinaus, daß das Überwinden räumlicher Grenzen und somit das Gewinnen neuer Geschäftspartner wichtige Schlüsselfaktoren bei der Internet-Nutzung sind. Diese Faktoren lassen sich wiederum über das Erschließen neuer Märkte (statistischer Zusammenhang zwischen "Neue Geschäftspartner gewonnen" und "Neue Märkte erschlossen" größer als 0,4) sowie dem Erreichen von Marktvorteilen (statistischer Zusammenhang zwischen "Neue Märkte erschlossen" und "Marktvorteile erreicht" größer als 0,4) monetarisieren. Monetarisiert wurden diejenigen drei Nutzeffekte, die einerseits bei jeweils über $50 \%$ der Unternehmen, die diese Nutzeffekte beobachten, zu einer Umsatzsteigerung führten, und andererseits bei weniger als $15 \%$ keine Umsatzsteigerung bewirkten.

\section{Ausgewählte Ergebnisse multivariater Analysen}

In diesem Abschnitt soll untersucht werden, ob ein statistischer Zusammenhang zwischen Wirtschaftszweigen auf der einen Seite und realisierten Nutzeffekten bzw. Umsatzsteigerungen auf der anderen Seite besteht. Außerdem wird überprüft, ob die Struktur der WWW-Anwendungen Einfluß auf den erzielten Nutzen und den Umsatz der Unternehmen hat.

\subsection{Zusammenhang zwischen Wirtschaftszweig und Nutzeffekten}

Wenn man die 489 an der empirischen Studie beteiligten Unternehmen auf die Sektoren der Wirtschaft aufteilt, entfallen auf den

- primären Sektor (Land- und Forstwirtschaft): 0 Unternehmen (0 \%),

- sekundären Sektor (produzierendes Gewerbe: Industrie, Handwerk, Energiewirtschaft, Baugewerbe):

61 Unternehmen $(12,5 \%)$,

- tertiären Sektor (Handel, Dienstleistungen): 428 Unternehmen $(87,5 \%)$.

Nachfolgend werden mögliche statistische Zusammenhänge zwischen Wirtschaftszweigen und Nutzeffekten bzw. Umsatzsteigerungen untersucht. Die 61 Unternehmen des produzierenden Gewerbes (sekundärer Sektor) werden mit den 428 Unternehmen aus dem Bereich Handel und Dienstleistungen (tertiärer Sektor) 
verglichen. Die Aggregation wurde vorgenommen, um globale Aussagen in Bezug auf realisierte Umsatzsteigerungen und Nutzeffekte treffen zu können. Sie ist darüber hinaus geboten, da die Anzahlen der Unternehmen in manchen Branchen sehr klein sind und somit stärker differenzierte Aussagen statistisch nicht abgesichert werden könnten.

Tabelle 4 zeigt, mit welchen prozentualen Häufigkeiten ausgewählte Nutzeffekte von den Unternehmen in den beiden Sektoren realisiert wurden. (Es handelt sich dabei um die 15 am meisten genannten Effekte sowie um drei weitere, die im Internet-Kontext als interessant erscheinen: "Engere zwischenbetriebliche Zusammenarbeit realisiert", "Stärkere Einflußnahme auf andere Unternehmen realisiert" sowie "Funktionen zusammengefaßt".) Anhand der letzten Spalte ist zu erkennen, ob ein Nutzeffekt in einem der beiden Sektoren überdurchschnittlich häufig realisiert werden konnte.

Auffällig ist, daß Unternehmen aus dem Bereich Dienstleistungen und Handel die meisten der Nutzeffekte in Tabelle 4 weitaus häufiger verzeichneten als Unternehmen des produzierenden Gewerbes. Die Unterschiede kommen besonders stark bei "Neue Märkte erschlossen", "Neue Geschäftspartner gewonnen", "Datenübertragung in Papierform entfällt", "Differenzierung im Wettbewerb", "Kundenzufriedenheit erhöht", "Arbeitszeit eingespart", "Funktionen zusammengefaßt" sowie "Engere zwischenbetriebliche Zusammenarbeit realisiert" zum Ausdruck.

\begin{tabular}{|l|c|c|c|}
\hline \multicolumn{1}{|c|}{ Nutzeffekte } & $\begin{array}{c}\text { Produzie- } \\
\text { rendes } \\
\text { Gewerbe }\end{array}$ & $\begin{array}{c}\text { Dienstlei- } \\
\text { stungen und } \\
\text { Handel }\end{array}$ & $\begin{array}{c}\text { Prozentualer } \\
\text { Anteil über } \\
\text { alle Bran- } \\
\text { chen }\end{array}$ \\
\hline Image verbessert & $68,9 \%$ & $68,0 \%$ & $68,1 \%$ \\
\hline Kundenservice erhöht & $47,5 \%$ & $52,8 \%$ & $52,1 \%$ \\
\hline Neue Geschäftspartner gewonnen & $34,4 \%$ & $52,6 \%$ & $50,3 \%$ \\
\hline Datenübertragung in Papierform entfällt & $39,4 \%$ & $50,2 \%$ & $48,9 \%$ \\
\hline Differenzierung im Wettbewerb & $32,8 \%$ & $44,2 \%$ & $43,1 \%$ \\
\hline Räumliche Grenzen überwunden & $36,1 \%$ & $42,5 \%$ & $41,7 \%$ \\
\hline Schnelleres Reagieren auf Kundenwünsche & $34,4 \%$ & $40,0 \%$ & $39,7 \%$ \\
\hline Neue Märkte erschlossen & $19,7 \%$ & $41,1 \%$ & $38,4 \%$ \\
\hline Umfassendere Informationsbasis realisiert & $39,3 \%$ & $37,4 \%$ & $37,6 \%$ \\
\hline Kundenzufriedenheit erhöht & $24,6 \%$ & $37,9 \%$ & $36,2 \%$ \\
\hline Abläufe vereinfacht & $54,1 \%$ & $33,7 \%$ & $36,2 \%$ \\
\hline Arbeitszeit eingespart & $23,0 \%$ & $37,0 \%$ & $35,2 \%$ \\
\hline Marktvorteile erreicht & $23,0 \%$ & $36,0 \%$ & $34,4 \%$ \\
\hline Kundenbindung erhöht & $29,5 \%$ & $35,0 \%$ & $34,2 \%$ \\
\hline Flexibilität erhöht & $26,2 \%$ & $35,5 \%$ & $34,4 \%$ \\
\hline Engere zwischenbetriebliche Zusammenarbeit & $4,9 \%$ & $16,6 \%$ & $15,5 \%$ \\
\hline Stärkere Einflußnahme auf andere Unternehmen & $8,2 \%$ & $9,6 \%$ & $9,4 \%$ \\
\hline Funktionen zusammengefaßt & $3,3 \%$ & $16,8 \%$ & $7,0 \%$ \\
\hline
\end{tabular}

Tabelle 4: Anteile realisierter Nutzeffekte differenziert nach Sektoren 
Vor allem marktorientierte Nutzeffekte, d.h. Nutzeffekte, die auf zwischenbetrieblicher Ebene bzw. Marktebene liegen, sind bei den Unternehmen aus Dienstleistungen und Handel wesentlich stärker repräsentiert. Dagegen konnten die Unternehmen des produzierenden Gewerbes mit "Abläufe vereinfacht" und "Umfassendere Informationsbasis realisiert" zwei Nutzeffekte, die zur Unternehmensbzw. Bereichsebene gehören, häufiger als andere realisieren.

\subsection{Zusammenhang zwischen Wirtschaftszweig und Umsatz- steigerung}

In diesem Abschnitt soll die Fragestellung untersucht werden, inwieweit zwischen den Unternehmen des produzierenden Gewerbes und den Dienstleistungs- und Handelsunternehmen Unterschiede in Bezug auf realisierte Umsatzsteigerungen bestehen. Tabelle 5 zeigt, wie häufig jeweils eine Umsatzsteigerung aufgrund des Internet-Engagements realisiert bzw. nicht realisiert wurde oder noch keine Einschätzung möglich war. In der letzten Spalte sind die prozentualen Häufigkeiten aus "Umsatzsteigerung realisiert (1)" und "Umsatzsteigerung nicht realisiert (2)" in Relation gesetzt.

\begin{tabular}{|l|c|c|c|c|}
\hline \multicolumn{1}{|c|}{ Sektor } & $\begin{array}{c}\text { Umsatzsteig. } \\
\text { realisiert (1) }\end{array}$ & $\begin{array}{c}\text { Umsatzsteig. } \\
\text { nicht realisiert } \\
\text { (2) }\end{array}$ & $\begin{array}{c}\text { Ohne } \\
\text { Einschätzung }\end{array}$ & $\begin{array}{c}\text { Relation } \\
(\mathbf{1}):(2)\end{array}$ \\
\hline Produzierendes Gewerbe & $6,6 \%$ & $23,0 \%$ & $65,6 \%$ & $0,29: 1$ \\
\hline Dienstleistungen und Handel & $23,1 \%$ & $13,8 \%$ & $56,3 \%$ & $1,67: 1$ \\
\hline
\end{tabular}

Tabelle 5: Realisierte Umsatzsteigerungen differenziert nach Sektoren

Im Bereich Dienstleistungen und Handel konnten immerhin 23,1 \% der Unternehmen durch ihr Internet-Engagement eine Umsatzsteigerung realisieren. Bei $13,8 \%$ Unternehmen ohne Umsatzsteigerung entspricht dies einem Verhältnis von $1,67: 1$. Im produzierenden Gewerbe bejahten nur 6,6\% der Unternehmen die Frage nach einer realisierten Umsatzsteigerung. Ihnen stehen $23 \%$ ohne Umsatzsteigerung gegenüber (Relation 0,29:1).

Der große Anteil von Unternehmen, die sich zu Umsatzwirkungen noch nicht äußern konnten, hängt damit zusammen, daß es teilweise schwierig ist, Umsätze direkt einem Internet-Engagement zuzurechnen. So besteht vor allem beim Handel nicht digitalisierbarer Produkte die Möglichkeit, daß sich potentielle Kunden zwar im WWW über das Produktspektrum eines Unternehmens informieren, den Kauf jedoch auf konventionelle Weise vollziehen.

Wenngleich der große Anteil von Unternehmen in der Spalte "Ohne Einschätzung" zu bedenken ist, so sind die Unterschiede zwischen dem Dienstleistungsund Handelssektor und dem Produktionssektor doch augenfällig. Teilweise können die Unterschiede damit erklärt werden, daß viele WWW-Angebote aus dem Dienstleistungs- und Handelssektor dem Business-to-Consumer-Bereich zuzurechnen sind. Das Potential zu direkten Umsatzsteigerungen ist hier größer, da die Unternehmen das Internet gezielt als weiteren Vertriebskanal nutzen können. Dies gilt um so mehr, je höher der Anteil digitalisierbarer Produkte ist. 


\subsection{Auswirkungen der Struktur von WWW-Anwendungen}

Unter strukturellen Gesichtspunkten wurden betriebliche WWW-Anwendungen an anderer Stelle nach den Kriterien Interaktivität, Reichweite und Automatisierungsgrad klassifiziert (vgl. Kurbel 1997; Kurbel 1998c). Aufbauend auf diesen Kriterien lassen sich Kategorien von WWW-Anwendungen mit gemeinsamen Merkmalen definieren. Die Kategorien unterscheiden sich unter anderem darin, in welchem Ausmaß das Internet zur Unterstützung der Geschäftsprozesse eingesetzt wird. Sie reichen von der passiven Informationsbereithaltung über interaktive Vorgangsabwicklung bis hin zur Automatisierung zwischenbetrieblicher Geschäftsprozesse und der Informationskooperation (z.B. virtuelle Unternehmen).

Die Kategorien wurden eingeführt, da allgemein akzeptierte Taxonomien des heutigen WWW-Angebots fehlen. Sieht man einmal von den technisch orientierten Einteilungen ab, so sind die bekannten betriebswirtschaftlich motivierten Klassifikationen eher pragmatisch fundiert oder nicht operational. So werden etwa Schlagwörter aus dem einschlägigen Sprachgebrauch wie "Elektronischer Prospekt", "Elektronischer Katalog" etc. (z.B. in Hansen 1996, S. 132 ff.), betriebliche Funktionen wie "Marketing", "Vertrieb", "Kundendienst" etc. (vgl. Renner 1996, 2 f.) oder Stufen der Porter'schen Wertschöpfungskette (vgl. Porter 1992, S. 59 ff.) zur Untergliederung verwendet (z.B. in Griese 1996, S. 11 ff.; Cronin 1994, S. 55 ff. und Alpar 1996, 171 ff.).

Von den 14 Haupt- und Unterkategorien, die in Kurbel 1998c definiert sind, wiesen sieben in der Untersuchung Einträge auf. Die meisten Unternehmen (60\%) ordneten sich der Kategorie "Informationsbereitstellung mit Kontaktangebot" zu (typisches Szenario: Unternehmensdarstellung, E-Mail-Link zur elektronischen Kontaktaufnahme), während höhere Kategorien nur schwach oder gar nicht besetzt sind (vgl. Kurbel/Teuteberg 1997, S. 10 f.). Im folgenden wird untersucht, ob ein Zusammenhang zwischen den Kategorien und einer Umsatzsteigerung bzw. den Nutzeffekten durch das entsprechende WWW-Angebot besteht.

\subsubsection{Zusammenhang zwischen Kategorien und Umsatzsteigerung}

Tabelle 6 zeigt, wie häufig in den sieben Kategorien mit Nennungen eine Umsatzsteigerung realisiert bzw. nicht realisiert werden konnte. In der letzten Spalte wurden die beiden Angaben jeweils in Relation gesetzt. So konnte z.B. durch WWW-Anwendungen der Kategorie "Anstoßen eines Vorgangs - automatische Bearbeitung" in 36,6 \% der Fälle eine Umsatzsteigerung realisiert werden; lediglich $4,9 \%$ in dieser Kategorie verzeichneten keine Umsatzsteigerung. Die Relation beträgt somit 7,5:1.

Sowohl der prozentuale Anteil derjenigen WWW-Anwendungen, mit denen eine Umsatzsteigerung realisiert werden konnte, als auch die Relation aus (1) und (2) steigen von der untersten Kategorie, "Informationsbereitstellung ohne Kontaktangebot", bis hin zur Kategorie "Interaktive Vorgangsabwicklung - manuelle Bearbeitung" kontinuierlich an. Daraus kann man schließen, daß mit zunehmenden Grad der WWW-Nutzung auch Umsatzzuwächse erzielt werden, d.h. je stärker 
das WWW-Angebot in die Geschäftsprozesse eingebunden ist, um so mehr profitieren die Unternehmen durch höheren Umsatz.

\begin{tabular}{|l|c|c|c|c|}
\hline \multicolumn{1}{|c|}{$\begin{array}{c}\text { WWW-Anwendungen } \\
\text { der Kategorie }\end{array}$} & $\begin{array}{c}\text { Umsatzsteig. } \\
\text { realisiert (1) }\end{array}$ & $\begin{array}{c}\text { Umsatzsteig. } \\
\text { nicht reali- } \\
\text { siert (2) }\end{array}$ & $\begin{array}{c}\text { Ohne Ein- } \\
\text { schätzung }\end{array}$ & $\begin{array}{c}\text { Relation } \\
\text { (1) : (2) }\end{array}$ \\
\hline $\begin{array}{l}\text { Informationsbereitstellung ohne } \\
\text { Kontaktangebot }\end{array}$ & 0,0 & 0,0 & 100,0 & $0: 0$ \\
\hline $\begin{array}{l}\text { Informationsbereitstellung mit } \\
\text { Kontaktangebot }\end{array}$ & 24,5 & 17,2 & 58,3 & $1,4: 1$ \\
\hline $\begin{array}{l}\text { Anstoßen eines Vorgangs - } \\
\text { manuelle Bearbeitung }\end{array}$ & 29,5 & 20,5 & 50,0 & $1,4: 1$ \\
\hline $\begin{array}{l}\text { Anstoßen eines Vorgangs - } \\
\text { automatische Bearbeitung }\end{array}$ & 36,6 & 4,9 & 58,5 & $7,5: 1$ \\
\hline $\begin{array}{l}\text { Interaktive Vorgangsabwicklung } \\
\text { - manuelle Bearbeitung }\end{array}$ & 100,0 & 0,0 & 0,0 & $100: 0$ \\
\hline $\begin{array}{l}\text { Interaktive Vorgangsabwicklung } \\
\text { - automatische Bearbeitung }\end{array}$ & 26,7 & 20,0 & 53,3 & $1,3: 1$ \\
\hline $\begin{array}{l}\text { Internet-Geschäftsprozeß- } \\
\text { schnittstellen - überwiegend } \\
\text { automatische Bearbeitung }\end{array}$ & 0,0 & 0,0 & 100,0 & $0: 0$ \\
\hline
\end{tabular}

Tabelle 6: Zusammenhang zwischen Kategorien und Umsatzsteigerung

In den oberen Kategorien sind Schlußfolgerungen allerdings nur noch bedingt möglich, da die Anzahl der Nennungen zu niedrig sind. Den Kategorien "Interaktive Vorgangsabwicklung - manuelle Bearbeitung" und "Internet-Geschäftsprozeßschnittstellen - überwiegend automatische Bearbeitung" ordnete sich nur jeweils ein Unternehmen zu, während es bei "Interaktive Vorgangsabwicklung - automatische Bearbeitung" immerhin noch 15 sind.

Auf soliderem Fundament steht die Einschätzung, daß sich durch stärkere Internet-Nutzung vermehrt Umsatzsteigerungen erzielen lassen, dagegen bei den tieferen Kategorien. Bemerkenswert sind die Verbesserungen durch zusätzliche Automatisierung, die beim Vergleich der beiden Kategorien "Anstoßen eines Vorgangs" (mit manueller bzw. automatischer Bearbeitung) erkennbar werden. Wenn die über das Internet angestoßenen Vorgänge (z.B. Bestelleingang) im Haus gleich automatisch weiterverarbeitet werden, steigt die Relation zwischen erreichten und nicht erreichten Umsatzsteigerungen von $1,4: 1$ auf $7,5: 1$.

\subsubsection{Zusammenhang zwischen Kategorien und Nutzeffekten}

Auch die Auswertung der Zusammenhänge zwischen Kategorien und Nutzeffekten läßt weitgehend nur Tendenzaussagen zu. In Tabelle 7 sind die prozentualen Häufigkeiten, mit denen ausgewählte Nutzeffekte in WWW-Anwendungen der verschiedenen Kategorien auftraten, wiedergegeben. Beispielsweise wurde der Nutzeffekt "Schnelleres und flexibleres Reagieren auf Kundenwünsche ermöglicht" bei keiner WWW-Anwendung der Kategorie "Informationsbereitstellung 
ohne Kontaktangebot", aber bei 73,3 \% der WWW-Anwendungen in Kategorie "Interaktive Vorgangsabwicklung - automatische Bearbeitung" realisiert.

Ein signifikantes Ansteigen der Nennungen mit höheren Kategorien läßt sich nur bei einigen Nutzeffekten ausmachen. Dies gilt insbesondere für die Effekte:

- "Datenübertragung in Papierform entfällt",

- "Umfassendere Informationsbasis realisiert",

- "Kundenzufriedenheit erhöht",

- "Abläufe vereinfacht".

\begin{tabular}{|c|c|c|c|c|c|c|c|}
\hline Kategorien & 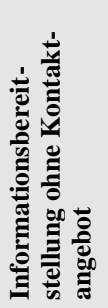 & 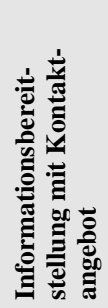 & 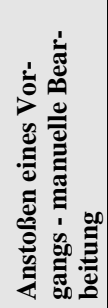 & 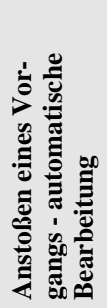 & 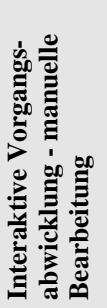 & 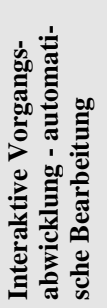 & 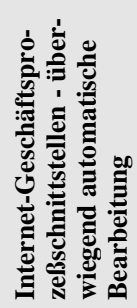 \\
\hline Image verbessert & 100,0 & 74,5 & 56,8 & 78,0 & 100,0 & 73,3 & 100,0 \\
\hline $\begin{array}{l}\text { Datenübertra- } \\
\text { gung in Papier- } \\
\text { form entfällt }\end{array}$ & 33,3 & 50,3 & 50,0 & 51,2 & 100,0 & 66,7 & 100,0 \\
\hline $\begin{array}{l}\text { Schnelleres Rea- } \\
\text { gieren auf Kun- } \\
\text { denwünsche }\end{array}$ & 0,0 & 40,7 & 40,9 & 51,2 & 0,0 & 73,3 & 0,0 \\
\hline $\begin{array}{l}\text { Umfassendere } \\
\text { Informations- } \\
\text { basis }\end{array}$ & 33,3 & 40,7 & 38,6 & 39,0 & 100,0 & 60,0 & 100,0 \\
\hline $\begin{array}{l}\text { Kundenzufrie- } \\
\text { denheit erhöht }\end{array}$ & 33,3 & 33,1 & 36,4 & 56,1 & 100,0 & 60,0 & 100,0 \\
\hline $\begin{array}{l}\text { Abläufe verein- } \\
\text { facht }\end{array}$ & 33,3 & 35,5 & 40,9 & 41,5 & 100,0 & 46,7 & 100,0 \\
\hline $\begin{array}{l}\text { Flexibilität er- } \\
\text { höht }\end{array}$ & 66,7 & 35,5 & 34,1 & 48,8 & 100,0 & 46,7 & 0,0 \\
\hline
\end{tabular}

Tabelle 7: Zusammenhang zwischen Kategorien und ausgewählten Nutzeffekten

Die prozentuale Häufigkeit von "Kundenzufriedenheit erhöht" steigt z.B. von 33,1 $\%$ in den beiden Kategorien "Informationsbereitstellung ..." auf 36,4 \% in der Kategorie "Anstoßen eines Vorgangs - manuelle Bearbeitung" und 56,1 \% in "Anstoßen eines Vorgangs - automatische Bearbeitung" an. In der noch mit 15 Anwendungen besetzten Kategorie "Interaktive Vorgangsabwicklung - automatische Bearbeitung" wurde die erhöhte Kundenzufriedenheit in $60 \%$ der Fälle registriert. Daß nicht bei mehr Nutzeffekten ein expliziter Nachweis über vermehrten Nutzen durch stärkeren WWW-Einsatz möglich ist, kann verschiedene Ursachen haben. In der empirischen Untersuchung wurden z.B. häufig organisatorische Probleme bei der Einbindung des WWW in die Geschäftsprozesse genannt (vgl. Kurbel 1997a). Darüber hinaus wurden die Ausprägungen der Nutzeffekte nur binär gemessen (realisiert/nicht realisiert). 
Ein Nutzeffekt wie "Schnelleres und flexibleres Reagieren auf Kundenwünsche ermöglicht" könnte aber in WWW-Anwendungen der einen Kategorie vielleicht "kaum", in Anwendungen einer anderen Kategorie dagegen "sehr stark" realisiert worden sein. In Tabelle 7 werden für diesen Effekt z.B. sowohl in der Kategorie "Informationsbereitstellung mit Kontaktangebot" als auch in der Kategorie "Anstoßen eines Vorgangs - manuelle Bearbeitung" jeweils ca. $41 \%$ ausgewiesen, obwohl die Annahme durchaus plausibel erscheint, daß in der letzteren die Reaktionsmöglichkeiten besser als in der ersteren ausgeprägt sind.

\section{Zusammenfassung}

Auf Basis der empirischen Analysen wurde gezeigt, daß Unternehmen, die im Internet präsent sind, Nutzeffekte realisieren, die über Wirkungsketten zu Umsatzsteigerungen führen. Zahlreiche Nutzeffekte sind kunden- und marktorientiert und haben wettbewerbsrelevante Wirkungen.

Die Nutzeffekte stehen über verschiedene Ebenen hinweg in einem Wirkungsgeflecht. Zwölf der fünfzehn am häufigsten realisierten Nutzeffekte stehen in einem starken statistischen Zusammenhang mit den Nutzeffekten "Marktvorteile erreicht" und "Kundenzufriedenheit erhöht". Exemplarisch wurde gezeigt, wie sich Nutzeffekte über verschiedene Ebenen hinweg monetarisieren lassen. 62,8\% bzw. $52,2 \%$ der Unternehmen, welche die beiden genannten Nutzeffekte beobachteten, konnten durch ihr Internet-Engagement eine Umsatzsteigerung erreichen.

Neben den kunden- und marktbezogenen Faktoren wurden die Nutzeffekte "Umfassendere Informationsbasis realisiert", "Flexibilität" bzw. "Lieferflexibilität erhöht" sowie "Räumliche Grenzen überwunden" und "Neue Geschäftspartner gewonnen" als wichtige Faktoren für Umsatzsteigerungen identifiziert.

Tendenziell gilt, daß in der empirischen Untersuchung Unternehmen des tertiären Sektors (Dienstleistungen und Handel) durch Nutzeffekte und Umsatzsteigerungen stärker profitieren als Unternehmen des sekundären Sektors (produzierendes Gewerbe). Intensivere Internet-Nutzung, wie sie den höheren der in Abschnitt 4.3 diskutierten Kategorien zugrunde liegt, verspricht mehr Umsatzsteigerung als die heute noch vorherrschende, relative passive Nutzung. Einschränkend ist festzuhalten, daß ein hoher Prozentsatz der Unternehmen in der Untersuchung sich zu Umsatzwirkungen des Internet-Engagements noch nicht äußern konnte. 


\section{Literaturverzeichnis}

Alpar, P. (1996): Kommerzielle Nutzung des Internet - Unterstützung von Marketing, Produktion, Logistik und Querschnittsfunktionen durch Internet, Intranet und kommerzielle Online-Dienste, 2. Auflage. Berlin, Heidelberg 1998.

Anderson, J.C./Gerbing, D.W. (1988): Structural equation modeling in practice: A review and recommended two-step approach. Psychological Bulletin 103 (1988) 3, S. 411-423.

Cronin, M.J. (1994): Doing Business on the Internet - How the Electronic Highway is Transforming American Companies. New York 1994.

Griese, J. (1996): Unternehmerische Konzepte zur Nutzung des Internet. In: Griese, J., Sieber, P. (Hrsg.), Internet - Nutzung für Unternehmungen. Bern u.a. 1996, S. 9-24.

Hansen, H.R. (1996): Klare Sicht am Info-Highway - Geschäfte via Internet \& Co. Wien 1996.

Hoffman, D.L., Novak, T.P., Chatterjee, P. (1995): Commercial Scenarios for the Web: Opportunities and Challenges; Journal of Computer-Mediated Communication, Special Issue on Electronic Commerce 1 (1995) 3; im WWW unter http://shum.huji.ac.il/jcmc/vol1/issue3/hoffman.html.

Janssen, J./Laatz, W. (1997): Statistische Datenanalyse mit SPSS für Windows, 2. Auflage. Berlin, Heidelberg 1997.

Kurbel, K. (1997): Internet-Nutzung im Business-to-Business-Bereich - Stand der Entwicklung, Typologie und Anwendungsbeispiele. In: Krallmann, H. (Hrsg.), Wirtschaftsinformatik '97 - Internationale Geschäftstätigkeit auf der Basis flexibler Organisationsformen und leistungsfähiger Informationssysteme. Heidelberg 1997, S. 23-34.

Kurbel, K. (1998a): Nutzeffekte und Hemmnisse der Internet-Nutzung durch deutsche Unternehmen. Industrie Management 14 (1998) 1, S. 9-13.

Kurbel, K. (1998b): Stand der Internet-Nutzung durch deutsche Unternehmen eine empirische Untersuchung. Industrie Management 14 (1998) 3, S. 7075 .

Kurbel, K. (1998c): Eine Datenbank mit innovativen Business-to-Business-Anwendungen im World Wide Web. In: Reichmann, T. (Hrsg.), Handbuch Globale Datennetze - Innovative Potentiale für Informationsmanagement und Controlling. München 1998, S. 39-58.

Kurbel, K./Teuteberg, F. (1997): Betriebliche Internet-Nutzung in der Bundesrepublik Deutschland - Ergebnisse einer empirischen Untersuchung. Arbeitsbericht, Lehrstuhl für Wirtschaftsinformatik, Europa-Universität Viadrina Frankfurt (Oder) 1997.

Kurbel, K./Teuteberg, F. (1998): The Current State of Business Internet Use: Results from an Empirical Study of German Companies. In: Baets, W.R.J. 
(Ed.), Proceedings of the 6th European Conference on Information Systems, Aix-en-Provence, France 1998, S. 542-556.

Linß, H. (1995): Integrationsabhängige Nutzeffekte der Informationsverarbeitung - Vorgehensmodell und empirische Ergebnisse. Wiesbaden 1995.

Porter, M.E. (1992): Wettbewerbsvorteile (Competitive Advantage) - Spitzenleistungen erreichen und behaupten, 3. Auflage. Frankfurt am Main 1992.

Renner, T. (1996): Kommerzielle Internetnutzung und Sicherheitsaspekte; in: Tagungsband Forum Online Publishing, Fraunhofer-Institut für Arbeitswissenschaft und Organisation, Stuttgart, 17. Juni 1996; http://www.mz.iao. fhg.de/event/op/renner.html.

Schinzer, H. (1998): Potentiale von Electronic Commerce für Unternehmen. CommerceNet Deutschland e.V., 1998.

Schumann, M. (1992a): Betriebliche Nutzeffekte und Strategiebeiträge der großintegrierten Informationsverarbeitung. Berlin u.a. 1992.

Schumann, M. (1992b): Wirtschaftlichkeitsrechnung für DV-Systeme. In: Huch, B. u.a. (Hrsg.), EDV-gestützte Controlling-Praxis. Frankfurt am Main 1992, S. 161-178.

Schumann, M./Mertens, P. (1990): Nutzeffekte von CIM-Komponenten und Integrationskonzepten (Teil 1). CIM Management 6 (1990) 3, S. 45-51.

Statistisches Bundesamt (Hrsg.) (1997): Statistisches Jahrbuch 1997 für die Bundesrepublik Deutschland. Wiesbaden 1997.

Teuteberg, F. (1998): Internet-Engagement deutscher Unternehmen - Plattform für Geschäfte. Gateway (1998) 5, S. 70-72. 